\title{
ON A SINGULAR ELLIPTIC EQUATION
}

\author{
WEI-MING NI ${ }^{1}$
}

\begin{abstract}
In this paper, we study the singular elliptic equation $L u+K(x) u^{p}=0$, where $L$ is a uniformly elliptic operator of divergence form, $p>1$ and $K(x)$ has a singularity at the origin. We prove that this equation does not possess any positive (local) solution in any punctured neighborhood of the origin if there exist two constants $C_{1}, C_{2}$ such that $C_{1}|x|^{\sigma} \geqslant K(x) \geqslant C_{2}|x|^{\sigma}$ near the origin for some $\sigma \leqslant-2$ (with no other condition on the gradient of $K$ ). In fact, an integral condition is derived.
\end{abstract}

1. Introduction. In recent years, there has been some interest in studying the following singular nonlinear elliptic equation

$$
\Delta u+h(x) u^{p}=0
$$

near the origin in $\mathbf{R}^{n}, n \geqslant 3$, where $\Delta=\sum_{i=1}^{n} \partial^{2} / \partial x_{i}^{2}, p>1$, and $h$ is nonnegative which may have the origin as an isolated singularity (see, for example, $[\mathbf{A}],[\mathbf{G}, \mathbf{S}],[\mathbf{L}]$ and the references therein). In $[\mathbf{A}],[\mathbf{G}, \mathbf{S}], h$ is assumed to satisfy the following conditions (in addition to being positive) near the origin.

$$
\begin{gathered}
C_{1}|x|^{\sigma} \geqslant h(x) \geqslant C_{2}|x|^{\sigma} \quad \text { for some constants } C_{1}, C_{2}>0 \text { and } \sigma \in \mathbf{R}, \\
|\nabla \log h(x)| \leqslant C_{3} /|x| \text { for some constant } C_{3}>0 .
\end{gathered}
$$

Indeed, the asymptotic behavior of positive singular solutions of (1) near the origin are obtained in case $\sigma>-2$ and $p$ in some appropriate ranges (cf. $[\mathbf{A}],[\mathbf{G}, \mathbf{S}]$ and $[\mathbf{L}])$. It is also shown in $[\mathbf{G}, \mathbf{S}]$ that if $\sigma<-2, h$ satisfies (2) and (3) and $1<p$ $<(n+2) /(n-2)$, then equation (1) does not possess any positive solution in $\Omega \backslash\{0\}$, where $\Omega$ is any neighborhood of the origin. The purpose of this present note is to show that equation (1) does not possess any positive solution in $\Omega \backslash\{0\}$ for any $p>1$ provided $h$ satisfies (2) and $\sigma \leqslant-2$, i.e. we obtain the same conclusion for all $p>1$ without any condition on $\nabla h$. Moreover, the exponent $\sigma \leqslant-2$ is best possible (cf. [A]). In fact, this follows from our main result which applies to more general elliptic operators than Laplacian and the condition on $h$ which insures the above conclusion is an integral condition which actually allows $h$ being "oscillatory" near the origin. We also mention that our proof is simple and elementary in contrast to the complicated proof in $[\mathbf{G}, \mathbf{S}]$.

Received by the editors October 18, 1982.

1980 Mathematics Subject Classification. Primary $35 \mathrm{~J} 60$.

Key words and phrases. Singular elliptic equation, nonexistence.

'Partially supported by NSF Grant MCS- 8200033 and a Summer Research Appointment from the Graduate School of the University of Minnesota. 
2. The main result. The equation we are studying is

$$
L u+K(x) u^{p}=0
$$

in $\Omega \backslash\{0\}$, where $\Omega$ is an arbitrary neighborhood of the origin in $\mathbf{R}^{n}, n \geqslant 3 ; p>1$, $K>0$ is smooth in $\Omega \backslash\{0\}$ and

$$
L \equiv \sum_{i, j=1}^{n} \frac{\partial}{\partial x_{i}}\left(a_{i j} \frac{\partial}{\partial x_{j}}\right)
$$

where $a_{i j} \in C^{2}(\Omega)$, is uniformly elliptic in $\Omega$ (we should point out that the conditions on $\left(a_{i j}\right)$ may be relaxed without causing any further work later on; see, for example, $\S 2$ in $[\mathbf{A}])$.

Let $G(x)$ be the Green's function of $L$ on $\Omega$ with singularity at the origin; we then define

$$
\tilde{K}(t)=\operatorname{Min}_{x \in \Gamma(t)} K(x)
$$

where $\Gamma(t)=\{x \in \Omega \mid G(x)=t\}$. Now, we can state our main result.

THEOREM. Under the above hypotheses on $L$ and $K$ equation (4) does not possess any positive solution in any punctured neighborhood of the origin provided

$$
\int^{\infty} t^{-n /(n-2)} \tilde{K}(t) d t=\infty
$$

Proof. The proof of our theorem uses some averaging method and a result in nonlinear oscillation theory for ordinary differential inequalities in recent papers of $\mathrm{Ni}[\mathbf{N}]$ and of Aviles $[\mathbf{A}]$. We define

$$
\bar{u}(t)=\int_{\Gamma(t)} \frac{\sum_{i, j=1}^{n} a_{i j} G_{x_{i}} G_{x_{j}}}{|\nabla G(x)|} u(x) d S
$$

as in $[\mathbf{A}]$. Then differentiating $\bar{u}$ twice with respect to $t$, applying Green's theorem several times, we arrive at the following inequality,

$$
\bar{u}_{t t}+C \frac{\tilde{K}(t)}{t^{2(n-1) /(n-2)}} \bar{u}^{p}(t) \leqslant 0
$$

for $t \geqslant T$, where $C$ is some (fixed) positive constant (see Lemma 1 in $\$ 2$ of [A] for details). Now, applying Theorem 3.43 in [N] to (6), we conclude our proof. Q.E.D.

REMARKS. (7) Since $G(x)$ behaves like $C /|x|^{n-2}$ near the origin (by the assumption on $L$ ), we see that (5) is fulfilled if $K(x)$ behaves like $|x|^{\sigma}, \sigma \leqslant-2$, near $x=0$. In fact, since only the total "weight" of $K$ matters, $K$ does not have to have $\infty$ as its limit at $x=0$, it could have zero as its limit inf at the origin.

NOTE ADDED IN PROOF. The conclusion of Theorem 3.43 in [N] is not quite correct as it stands, what is really proved there is that a solution cannot be ultimately positive, which is just sufficient for the application in $[\mathbf{N}]$ and is just what we need in the proof of the main result here. In fact this result in nonlinear oscillation was proved earlier by $\mathrm{H}$. Teufel, Jr., $A$ note on second-order differential equations and functional 
differential equations, appeared in Pacific J. Math. 41 (1972), 537-541, and generalized by F. V. Atkinson, see, e.g. On second-order differential inequalities, in Proc. Roy. Soc. Edinburgh (A) 72 (1973), 109-127. The author wishes to thank Professor Atkinson for pointing out these remarks.

\section{REFERENCES}

[A] P. Aviles, On isolated singularities in some nonlinear partial differential equations, Indiana Univ. Math. J. (to appear).

[G,S] B. Gidas and J. Spruck, Global and local hehavior of positive solutions of nonlinear elliptic equations, Comm. Pure Appl. Math. 36 (1981), 525-598.

[L] P.-L. Lions, Isolated singularities in semilinear problems, J. Differential Equations 38 (1980), 441-450.

[N] W.-M. Ni, On the elliptic equation $\Delta u+K(x) u^{(n+2) /(n-2)}=0$, its generalizations and applications in geometry, Indiana Univ. Math. J. 31 (1982), 493-529.

School of Mathematics, University of Minnesota, Minneapolis, Minnesota 55455 